CLINICAL STUDY

\title{
Kinetics of removal of intravenous testosterone pulses in normal men
}

\author{
Johannes D Veldhuis, Daniel M Keenan ${ }^{1}$, Peter Y Liu and Paul Y Takahashi ${ }^{2}$ \\ Endocrine Research Unit, Mayo School of Graduate Medical Education, Clinical Translational Science Center, Mayo Clinic, Rochester, Minnesota 55905, \\ USA, ${ }^{1}$ Department of Statistics, University of Virginia, Charlottesville, Virginia 22904, USA and ${ }^{2}$ Department of Internal Medicine, Mayo School of \\ Graduate Medical Education, Clinical Translational Science Center, Mayo Clinic, Rochester, Minnesota 55905, USA \\ (Correspondence should be addressed to J D Veldhuis; Email: veldhuis.johannes@mayo.edu)
}

(P Y Liu is now at Endocrine and Metabolic Group, Department of Andrology, ANZAC Research Institute, Woolcock Institute of Medical Research, Concord Hospital, University of Sydney, Sydney, New South Wales 2139, Australia)

\begin{abstract}
Background: Testosterone is secreted into the bloodstream episodically, putatively distributing into total, bioavailable (bio) nonsex hormone-binding globulin (nonSHBG-bound), and free testosterone moieties. The kinetics of total, bio, and free testosterone pulses are unknown.

Design: Adrenal and gonadal steroidogenesis was blocked pharmacologically, glucocorticoid was replaced, and testosterone was infused in pulses in four distinct doses in 14 healthy men under two different paradigms (a total of 220 testosterone pulses).

Methods: Testosterone kinetics were assessed by deconvolution analysis of total, free, bioavailable, SHBG-bound, and albumin-bound testosterone concentration-time profiles.

Results: Independently of testosterone dose or paradigm, rapid-phase half-lives ( $\mathrm{min}$ ) of total, free, bioavailable, SHBG-bound, and albumin-bound testosterone were comparable at $1.4 \pm 0.22 \mathrm{~min}$ (grand mean \pm s.E.M. of geometric means). Slow-phase testosterone half-lives were highest for SHBGbound testosterone ( $32 \mathrm{~min}$ ) and total testosterone $(27 \mathrm{~min})$ with the former exceeding that of free testosterone (18 $\mathrm{min})$, bioavailable testosterone (14 $\mathrm{min})$, and albumin-bound testosterone (18 $\mathrm{min}$; $P<0.001)$. Collective outcomes indicate that i) the rapid phase of testosterone disappearance from point sampling in the circulation is not explained by testosterone dose; ii) SHBG-bound testosterone and total testosterone kinetics are prolonged; and iii) the half-lives of bioavailable, albumin-bound, and free testosterone are short.

Conclusion: A frequent-sampling strategy comprising an experimental hormone clamp, estimation of hormone concentrations as bound and free moieties, mimicry of physiological pulses, and deconvolution analysis may have utility in estimating the in vivo kinetics of other hormones, substrates, and metabolites.
\end{abstract}

European Journal of Endocrinology 162 787-794

\section{Introduction}

The availability of systemic testosterone and estradiol $\left(\mathrm{E}_{2}\right)$ to target organs depends upon glandular secretion into the bloodstream, transportation in plasma, entry into tissue fluids, retention at target cells, and metabolic transformation. Secretion of gonadal sex steroids proceeds via an admixture of continuous low basal release and superimposed bursts that reflect pulsatile gonadotropin drive (1-3). Pulsatile testosterone secretion has been corroborated by direct testicular-vein sampling in humans (4). In principle, a pulse of testosterone secreted into systemic blood diffuses within the aqueous compartment, distributes among plasma proteins, exits the vascular tree, and/or is degraded and transformed (5). Thus, gonadal secretion of a pulse of testosterone would be expected to yield time-varying concentrations of total, sex hormone-binding globulin (SHBG)-bound, albumin-bound, bioavailable (nonsex steroid-binding globulin (SHBG)-bound), and free (nonSHBG and nonalbumin-bound) testosterone. However, in species such as humans, high-affinity transport proteins exist in plasma, which greatly dampen pulsatile testosterone profiles making kinetic estimates more difficult (6-8). We postulated that if endogenous testosterone were depleted, infusion of (exogenous) testosterone pulses would allow one to directly calculate testosterone moiety-specific disappearance rates, thereby estimating physiological kinetics.

The present studies introduce a model for quantifying the dynamics of testosterone pulses in vivo. In particular, the paradigms comprise pharmacological inhibition of adrenal and testicular steroidogenesis at the level of cholesterol side-chain cleavage (CYP11A) with 
glucocorticoid addback (because of concomitant cortisol depletion), and i.v. infusion of dose-varying testosterone pulses either superimposed or not superimposed upon basal testosterone infusion. Total, bioavailable (bio), SHBG-bound, albumin-bound, and free testosterone concentrations were first estimated in successive 10-min serum samples using subject-specific measurements of SHBG and albumin. Deconvolution analysis was then applied to each train of moiety-defined testosterone pulses to quantify elimination kinetics. Thereby, we could assess the relative contributions of SHBG and albumin-bound testosterone moieties to total and free testosterone kinetics. The outcomes were similar in both paradigms, and therefore, they have relevance to understanding the physiology of regulated testosterone egress from the circulation.

\section{Methods}

\section{Subjects}

Fourteen healthy men aged 21-50 years (range) with body mass indices (BMI) of $19-31 \mathrm{~kg} / \mathrm{m}^{2}$ participated in the study. Volunteers were healthy, community-dwelling unmedicated men who provided written informed consent approved by the Mayo Institutional Review Board. The protocol was reviewed by the USA Food and Drug Administration. Outpatient history and physical examination excluded any recent medical illness; systemic disease; liver, renal, or hematological abnormalities; or concurrent drug use. Screening laboratory tests were normal, including baseline glucose, creatinine, hepatic transaminases, complete blood count, morning cortisol, LH, FSH, prolactin, TSH, total testosterone, insulin-like growth factor $1, \mathrm{E}_{2}$, SHBG, albumin, minerals, and electrolytes.

\section{Protocol}

In the first protocol, volunteers were admitted to the Mayo Clinic Translational Science Unit (CRU) on three separate randomly ordered evenings scheduled at least 2 weeks apart. At $2000 \mathrm{~h}$, an indwelling i.v. catheter was inserted in each forearm, and kept patent by saline infusion $(10 \mathrm{ml} / \mathrm{h})$. At $2400 \mathrm{~h}$, a constant i.v. infusion of testosterone, $1.7 \mu \mathrm{mol} / \mathrm{h}$, was begun and continued for $18.5 \mathrm{~h}$ ( $n=9$ subjects). The other i.v. catheter was used for 10-min blood sampling starting at $0500 \mathrm{~h}$ the next morning and continuing for $13.5 \mathrm{~h}$. testosterone pulses were injected starting at $0800 \mathrm{~h}$, one dose level $(0.46,1.4$, or $4.2 \mu \mathrm{mol} /$ bolus $)$ per admission. A pulse was delivered intravenously over $30 \mathrm{~min}$ every $90 \mathrm{~min}$ for a total of seven pulses. This schedule emulates the inferred pattern of endogenous testosterone secretion (9). In the second protocol, another cohort of five subjects received overnight saline infusion followed by nine consecutive 1-min i.v. bolus injections of
$1.7 \mu \mathrm{mol} /$ bolus crystalline testosterone beginning at $0800 \mathrm{~h}$. The rapid-bolus format mimics most earlier studies in pharmacology. Blood was sampled concurrently every $10 \mathrm{~min}$ for $13.5 \mathrm{~h}$. This protocol addition allowed us to assess whether half-life estimates are similar after 30 and 1-min bolus injections and in the presence and absence of basal testosterone infusion. Thus, 14 subjects were studied altogether. Testosterone infusions were prepared as described earlier. Three oral doses of ketoconazole, a steroidogenic inhibitor (10), were administered as follows: $1000 \mathrm{mg}$ at $2200 \mathrm{~h}$, $400 \mathrm{mg}$ at $0600 \mathrm{~h}$, and $400 \mathrm{mg}$ at $1200 \mathrm{~h}$. Dexamethasone $(0.75 \mathrm{mg})$ was given orally at $2200 \mathrm{~h}$ with the first ketoconazole dose and again $(0.5 \mathrm{mg})$ at the end of sampling, since ketoconazole blocks CYP11A and depletes cortisol (10). Dinner, lunch, and breakfast were provided. Lights were extinguished at $2230 \mathrm{~h}$. Ambulation was permitted within the sampling room. Alcohol use was disallowed.

\section{Sex steroid measurements}

Total testosterone was measured by immunochemiluminescence technology (ACS 180, Bayer; interassay coefficient of variation (CV) 6-11\%, lower limit of detection $0.17 \mathrm{nmol} / \mathrm{l})$. Cross-reactivity of this assay was $5 \%$ with $5 \alpha$-dihydrotestosterone and $<1 \%$ for all other testosterone metabolites. Total $\mathrm{E}_{2}$ was measured using a double-antibody RIA (Diagnostic Products Corporation, Los Angeles, CA, USA; interassay CV 4.5-8\%, lower limit of detection $18 \mathrm{pmol} / \mathrm{l})$. Cross-reactivity was $12 \%$ with estrone and $6 \%$ or less with other estrogen metabolites. Liquid chromatography-tandem mass spectrometry (LC-MS/MS, API 5000, Applied Biosystems-MDS Sciex, Foster City, CA, USA) was used for corroborative measurements in a set of 30 samples. Testosterone was prepared by acetonitrile precipitation and high-throughput LC extraction. Analysis was performed by MS/MS equipped with a heated nebulizer ion source. Deuterated $d_{3}$-testosterone served as an internal standard. Values as low as $0.035 \mathrm{nmol} / \mathrm{l}$ were detectable by this method. For testosterone values of $0.28,0.14,0.07$, and $0.035 \mathrm{nmol} / \mathrm{l}$, respective $\mathrm{CV}$ values were $7.5,2.2,6.3$, and $28.8 \%$. The coefficient of determination for total testosterone in the two assay systems was $R^{2}=0.95$ with a slope of 0.96 and $y$-intercept of $-0.347 \mathrm{nmol} / \mathrm{l}$.

Albumin was measured in serum collected hourly during the testosterone infusions by the Roche/Hitachi 912 System. SHBG was quantified in hourly samples using a chemiluminescence immunoassay (Diagnostic Products Corporation; interassay CV 4.8-8\%). The nonSHBG-bound (bioavailable) fraction of total testosterone and $\mathrm{E}_{2}$ was measured using a modification of the technique of O'Connor et al. (11) and Tremblay et al. Percentage bioavailable testosterone or $\mathrm{E}_{2}$ was multiplied by total testosterone or $\mathrm{E}_{2}$ determined by immunoassay or mass spectroscopy to obtain respective bioavailable 
testosterone or $\mathrm{E}_{2}$ concentrations. Free testosterone was estimated by equilibrium dialysis of undiluted plasma at $37{ }^{\circ} \mathrm{C}(12)$.

Other screening hormones were assayed as described (13).

\section{Calculation of bio and free testosterone concentrations}

Free and bio testosterone concentrations as well as SHBG- and albumin-bound testosterone concentrations were calculated in each 10-min serum sample using measured total testosterone concentrations, albumin, and SHBG (13). The equation system was adapted from Sodergard et al. (14). The association constants were estimated empirically for testosterone-SHBG and testosterone-albumin as $1.78 \times 10^{9}$ and $1.80 \times 10^{4} \mathrm{M}$ respectively based upon the optimization of the correlation between calculated and directly measured bio and free testosterone concentrations (see supplemental data in (13).

\section{Deconvolution analysis}

Testosterone concentration time series were analyzed using a recently developed automated deconvolution method. The algorithm was verified mathematically by direct statistical proof and validated empirically using hypothalamic-pituitary sampling and simulated pulsatile time series (15). First, the Matlab-based program (MathWorks, Inc., Natwick, MA, USA) detrends the data and normalizes concentrations to the unit interval $[0,1]$. Secondly, a smoothing process (a nonlinear adaptation of the heat-diffusion equation) creates multiple successively decremental potential pulse-time sets, each containing one fewer burst. Thirdly, a maximum-likelihood expectation estimation method computes all secretion and elimination parameters simultaneously conditional on each of the candidate pulse-time sets. Deconvolution parameters comprise basal secretion $\left(\beta_{0}\right)$, two half-lives $\left(\alpha_{1}\right.$ and $\left.\alpha_{2}\right)$, linear determinants of secretory-burst mass $\left(\eta_{0}\right.$ and $\left.\eta_{1}\right)$, random effects on burst mass $\left(\sigma_{\mathrm{A}}\right)$, procedural and measurement error $\left(\sigma_{\varepsilon}\right)$, and a three-parameter flexible Gamma secretory-burst waveform $\left(\beta_{1}, \beta_{2}\right.$, and $\left.\beta_{3}\right)$. The slow half-life of testosterone was represented as $63 \%$ of the decay amplitude (16). The fast and slow testosterone half-lives were estimated from the data simultaneously with the other parameters. Statistical model selection was performed to distinguish among the deconvolution fits of the candidate pulse-time sets using the Akaike information criterion (17). The deconvolution parameters (and units) reported here comprise fast and slow half-lives (min) and mass of testosterone infused per burst (concentration units). The apparent distribution volume is the dose infused divided by the calculated mass delivered per pulse.

\section{Statistical analysis}

Estimates of the deconvolution parameters were transformed to the natural logarithmic scale to produce symmetric distributions and equalize measurement variability. Logarithmic measurements were analyzed via mixed-effects two-way ANOVA for repeated measures. For each deconvolution variable, the ANOVA model specification included two classification factors to estimate the main effect of testosterone dose (four factors) and testosterone moiety (five factors). Model parameters were evaluated via residual maximum likelihood, and the variance-covariance matrix was modeled in the compound symmetry form (18). A priori comparisons were formulated by way of linear contrasts of the least-squares means. Tukey's honestly significant difference criterion was utilized to maintain an overall two-sided multiple-comparisons type I error of 0.05 . Half-life values are reported as the geometric mean (95\% confidence interval), and other data (including grand means) are reported as the arithmetic mean \pm s.E.M.

Linear regression was used to test for an effect of the three doses on testosterone half-life in the subjects so studied. Standardized slopes were tested against the null hypothesis of a zero-mean unit standard deviation distribution of $\mathrm{z}$ scores by the Kolmogorov-Smirnov statistic.

\section{Results}

Baseline hormone data in the 14 subjects included normal concentrations of LH (mean $4.2 \pm 0.5 \mathrm{IU} / \mathrm{l}$ ), FSH $(3.8 \pm 0.6 \mathrm{IU} / \mathrm{l})$, prolactin $(9.6 \pm 0.7 \mu \mathrm{g} / \mathrm{l})$, SHBG $(25 \pm 3.0 \mathrm{nmol} / \mathrm{l}), \mathrm{E}_{2}(107 \pm 11 \mathrm{pmol} / \mathrm{l})$, total testosterone $(18 \pm 1.3)$, bio testosterone $(5.2 \pm 0.87)$, and free testosterone $(0.52 \pm 0.073) \mathrm{nmol} / \mathrm{l}$. Two subjects dropped out for scheduling reasons before completing the $0.46 \mu \mathrm{mol}$ testosterone dose. Nine subjects completed both the 1.4 and $4.2 \mu \mathrm{mol}$ testosterone doses. Five other individuals received $1.7 \mu \mathrm{mol}$ testosterone as 1-min bolus injections, so as to compare kinetic estimates with those following the 30-min infusions.

Mean and peak testosterone concentrations, which were measured in serum collected every $10 \mathrm{~min}$ after $0.46,1.4$, and $4.2 \mu \mathrm{mol}$ testosterone injections given over $30 \mathrm{~min}$ during basal infusion, increased with testosterone dose $(P<0.001$ by one-way ANOVA). This was true for each of total, bio, free, SHBG-bound, and albumin-bound testosterone concentrations (Supplementary Table 1, see section on supplementary data given at the end of this article). Injection of $1.7 \mu \mathrm{mol}$ testosterone by 1 -min bolus with no basal testosterone infusion yielded mean and peak total testosterone concentrations lower than those observed after 1.4 as well as $4.2 \mu \mathrm{mol}$ testosterone given as 30-min pulses, indicating that the manner (not just 
the dose) of testosterone infusion influences testosterone concentrations. Peak testosterone concentrations exceeded the normal range after the highest testosterone dose. Time profiles of total, bio, and free testosterone are illustrated for all three testosterone pulse sizes in Fig. 1A, and for SHBG-bound and albumin-bound testosterone in Fig. 1B for one subject.

Two-way ANOVA in a $4 \times 5$-factor design was used to test the influence(s) of testosterone dose (four independent variables) and testosterone moiety (five types) on the rapid-phase testosterone half-life (dependent variable) (Supplementary Table 2, see section on supplementary data given at the end of this article). The testosterone-dose effect was significant $(P=0.007)$, but only due to a slight prolongation of the rapid testosterone half-life at a testosterone dose of $1.4 \mu \mathrm{mol}$ compared with 0.46 or $1.7 \mu \mathrm{mol}(P \leq 0.028)$. There was no effect of testosterone moiety and no dose $\times$ moiety interaction. Rapid-phase testosterone half-lives (min) averaged across all four testosterone doses are shown for each testosterone moiety in Fig. 2 (Panel A). The grand arithmetic mean \pm s.E.M. of (geometric mean) rapid-phase testosterone half-lives was $1.4 \pm 0.22 \mathrm{~min}$ for all five testosterone moieties and all four testosterone doses $(n=14$ subjects).
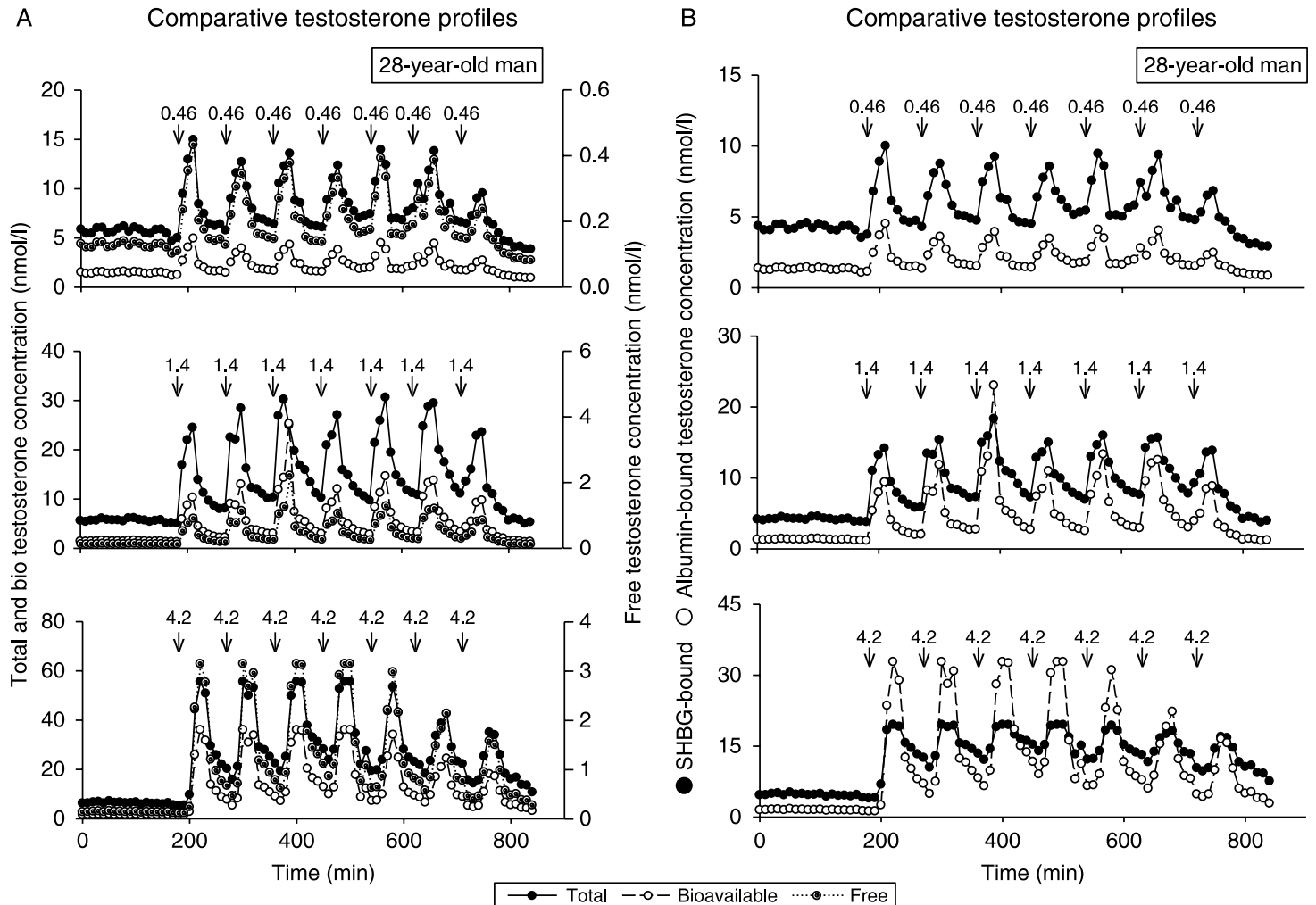

Figure 1 Illustrative pulsatile testosterone-concentration profiles in a 28-year-old subject administered ketoconazole and glucocorticoid replacement to deplete endogenous testosterone followed by seven consecutive 30 -min intravenous pulses of $0.46,1.4$, or $4.2 \mu \mathrm{mol}$ testosterone (top to bottom), one every 90 min superimposed upon a basal testosterone infusion, following a 3-h baseline interval. Panel A: profiles of three testosterone moieties (total, bioavailable, and free) estimated in each 10-min serum sample from mean SHBG and albumin concentrations measured every hour (Methods). Panel B: profiles of SHBG- and albumin-bound testosterone in the same subject.

www.eje-online.org 

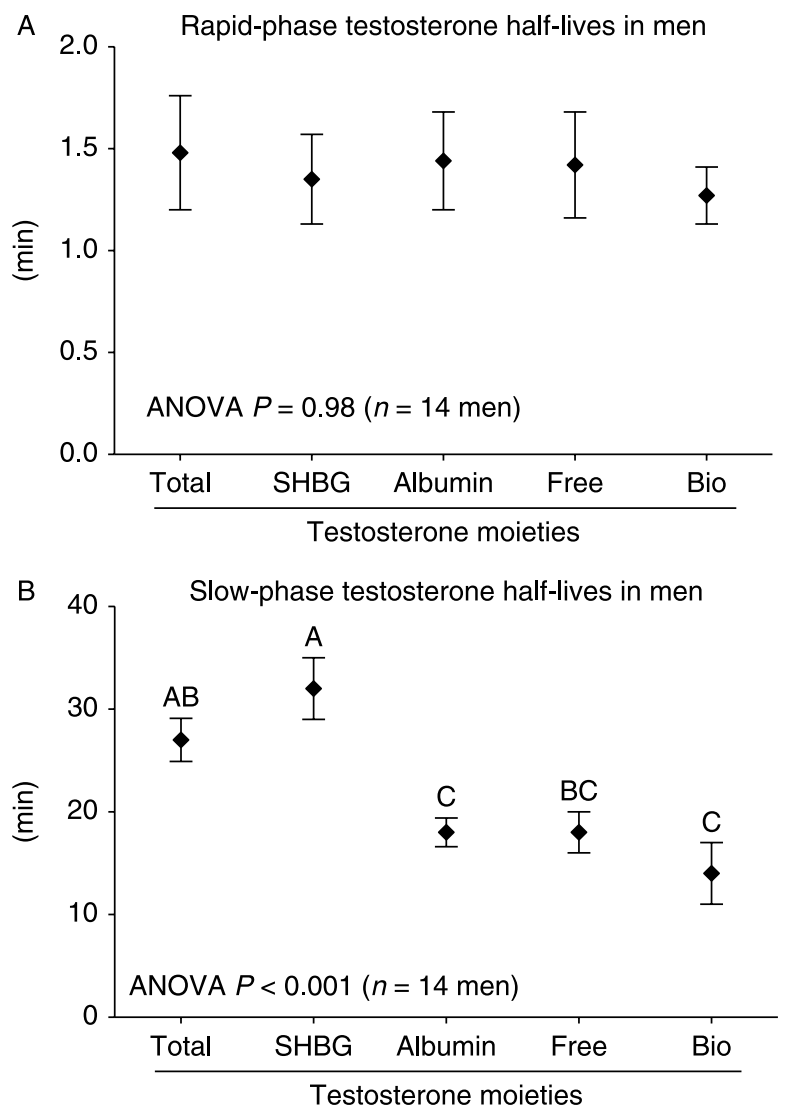

Figure 2 Deconvolution-estimated biexponential half-lives of moiety-specific testosterone pulses averaged across four testosterone doses in 14 men. Data are the arithmetic mean \pm S.E.M. (of geometic means for the individual four testosterone doses in Supplementary Tables 1 and 2) for the rapid (Panel A) and the slow (Panel B) phases of testosterone disappearance. $P$ values were estimated by ANOVA. Different means are denoted by unique (unshared) alphabetic characters (B).

Deconvolution estimates of the mass of testosterone infused per pulse per unit distribution volume are summarized in Supplementary Table 4 (see section on supplementary data given at the end of this article). Two-way ANOVA in a $4 \times 5$-factor design $(n=14$ subjects) of deconvolution-calculated testosterone pulse-mass values disclosed main effects of testosterone dose $(P<0.001)$ and testosterone moiety $(P<0.001)$, as well as a major interaction between the two factors $(P<0.001)$. With respect to testosterone dose, values for each of the four doses differed from those for each of the three others by post hoc Tukey's test $(P \leq 0.004)$. With respect to testosterone moiety, estimated (infused) free testosterone mass was less than that of all others $(P<0.001)$. Deconvolution-calculated mass values for albumin- and SHBG-bound testosterone were similar as were values for albumin-bound and bio testosterone at all testosterone doses.

The distribution volume $\left(V_{\mathrm{d}}\right)$ was calculated as the quotient of the known mass of testosterone injected per pulse ( $\mu \mathrm{mol})$ and the deconvolution-estimated mass of testosterone infused per pulse $(\mu \mathrm{mol} / \mathrm{l})$. Data were expressed as $1 / \mathrm{m}^{2}$ body surface. Calculated testosterone moiety-specific $V_{\mathrm{d}}$ values are given by dose in Fig. 3 for $0.46,1.4$, and $4.2 \mu \mathrm{mol}$ testosterone injections in the nine subjects given 30-min testosterone boluses. According to two-way ANOVA, dose of testosterone and testosterone moiety influenced apparent $V_{\mathrm{d}}$ (both $P<0.001$ main effects) with a weak interaction $(P=0.04)$. With respect to dose effects, calculated $V_{d}$ was about twofold greater for the $4.2 \mu \mathrm{mol}$ testosterone dose than for the $1.4 \mu \mathrm{mol}$ testosterone dose $(P<0.001)$. Estimated $V_{\mathrm{d}}$ values for the 1.4 and $0.46 \mu \mathrm{mol}$ testosterone doses were comparable for all moieties except for total and SHBG-bound testosterone. In relation to testosterone moieties, mean $V_{\mathrm{d}}$ for total testosterone was less than that for all other moieties (each $P<0.001) . V_{\mathrm{d}}$ for free testosterone was greater than that for all other moieties $(P<0.001)$. Post hoc testing by Tukey's procedure indicated that $V_{\mathrm{d}}$ estimates were similar for the following three pairs independently of testosterone dose: i) albumin- and SHBG-bound T; ii) bio and albumin-bound T; and iii) bio and SHBG-bound testosterone. In contrast, estimates of $V_{\mathrm{d}}$ made after $1.7 \mu \mathrm{mol}$ testosterone given by 1-min bolus injection without basal testosterone infusion $(n=5)$ were elevated by 2.5 - to 6 -fold over those after $4.2 \mu \mathrm{mol}$ testosterone given by 30-min infusion. An exception was SHBG-bound testosterone. In particular, 1-min bolus testosterone injections yielded $V_{\mathrm{d}}$ estimates for total, bio, free, SHBG-bound, and albumin-bound testosterone of respectively $102 \pm 17,391 \pm 111$, $4451 \pm 1265,145 \pm 17$, and $428 \pm 122 \mathrm{l} / \mathrm{m}^{2}$.

\section{Discussion}

Deconvolution analyses of 220 discrete pulses of testosterone delivered intravenously across four testosterone doses in 14 healthy men during pharmacological inhibition of adrenal and testicular steroidogenesis disclosed that rapid-phase testosterone half-lives are essentially independent of the duration of testosterone-infusion pulse (1 vs $30 \mathrm{~min}$ ), testosterone dose (ninefold range), plasma testosterone moiety (five moieties assessed) and a $>100$-fold range in peak testosterone concentrations. In contradistinction, estimated slow-phase half-lives were dependent upon testosterone moiety (total, free, bio, SHBG-bound, and albumin-bound) and testosterone dose pulse size (0.46, $1.4,1.7$, and $4.2 \mu \mathrm{mol}$ ). Two-way ANOVA disclosed that total and SHBG-bound testosterone half-lives are statistically comparable (grand mean $30 \pm 2.5$ min for $n=14$ men), whereas half-lives of free, albumin-bound, and bio testosterone are similar (grand mean $16 \pm 2.8 \mathrm{~min}$ ). The collective data indicate that estimated rapid and slow kinetics of total, bio, and free testosterone pulses have distinct dependencies upon SHBG and albumin. 


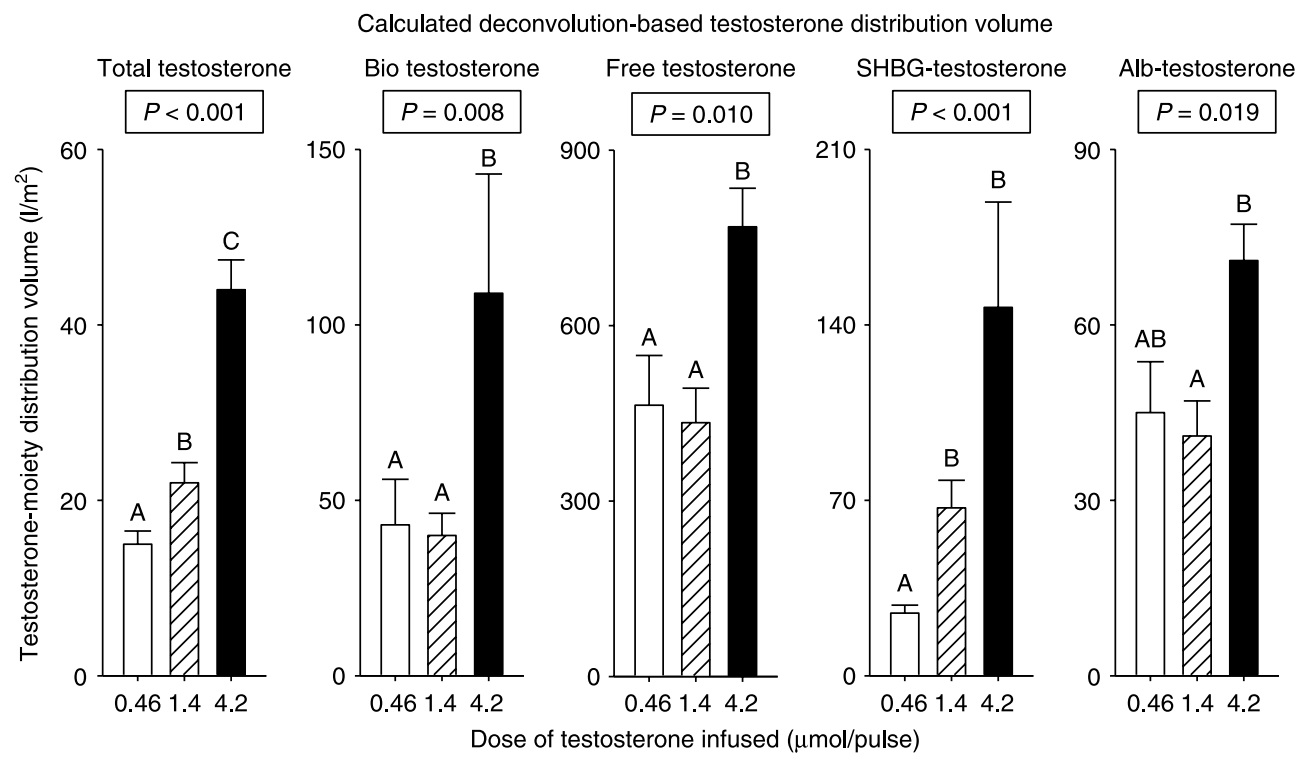

Figure 3 Distribution volumes $\left(1 / \mathrm{m}^{2}\right)$ of total, SHBG-bound, albumin-bound, free, and bioavailable testosterone in nine healthy young and middle-aged men given three different i.v. doses of testosterone as 30 -min pulses. To obtain $V_{d}$, the injected testosterone dose was divided by the deconvolution-estimated pulse mass (rather than by the peak testosterone concentration).

A grand mean rapid-phase testosterone half-life of $1.4 \pm 0.22$ min was estimated for total, SHBG-bound, free, bio, and albumin-bound testosterone. The consistency of individual estimates across testosterone moieties (Supplemental Table 2) suggests a common limiting step in rapid-phase testosterone disappearance, such as intravascular mixing by diffusion and advection (19). Another analytically based estimate of the rapid-phase half-life of total testosterone in 15 men was $2.8 \mathrm{~min}$, which was obtained indirectly without inhibiting endogenous testosterone production or injecting exogenous testosterone (3). Estimation of intravenous radiolabeled testosterone kinetics in five normal young men yielded a fast-phase half-life of total testosterone of $7.0 \mathrm{~min}$ (16). Our recalculation from the mean data in that study predicted a value of $4.95 \mathrm{~min}$. In a third study, $1 \mu \mathrm{mol}$ unlabeled testosterone was infused intravenously over $20 \mathrm{~min}$ in 11 men aged $72 \pm 5$ years in the presence of endogenous testosterone, yielding a rapid-phase total testosterone half-life of $7.5 \mathrm{~min}$ (20). The unlabeled testosterone infusion study did not take the precaution of depleting endogenous testosterone to avoid confounding by endogenous testosterone pulses, which predictively would artificially prolong the rapidphase half-life estimate. To our knowledge, no previous study has estimated the kinetics of SHBG-bound, albumin-bound, free, and bioavailable testosterone. Moreover, the many studies that infused testosterone continuously are restricted in physiological interpretation, since testosterone is normally secreted in pulses.

The grand mean slow-phase half-life of total testosterone was estimated here as $27 \pm 2.1 \mathrm{~min}$, from geometric mean values of $21,27,27$, and $31 \mathrm{~min}$ for respective testosterone doses of $0.46,1.4,1.7$, and
$4.2 \mu \mathrm{mol} /$ bolus. Linear regression analysis showed that testosterone dose did not correlate with slow-phase testosterone half-lives, but the study was not powered to test this idea. Earlier analytical model-based predictions of the slow-phase half-life of endogenously secreted total testosterone averaged about $45 \mathrm{~min}$ in healthy men (3). Our estimate at higher testosterone doses is similar to that inferred by Horton et al. after injecting a single dose of tritiated testosterone in five men, viz. 34 min (16), and is less than that calculated by White et al. after injecting a single dose of unlabeled testosterone in older eugonadal men, viz. 56 min (20). In addition to clear methodological differences, disparate estimates of the physiological range of total-testosterone kinetics could reflect i) unequal hepatic extraction of testosterone, ii) genetic variations in SHBG concentrations, and iii) variable activity of sex steroid-metabolizing cytochrome P450 enzymes (21-26). The first hypothesis reflects the major role of the liver in the transformation and excretion of testosterone. The second hypothesis is based upon the capability of SHBG, but not of albumin, infusion to prolong total testosterone half-life in animal models (26). Estrogen administration elevates SHBG concentrations and also prolongs the half-life of total testosterone in humans (25). The third hypothesis arises from known genetic polymorphisms in testosterone metabolism (23).

Our data support the possibility that SHBG-bound testosterone rather than albumin-bound testosterone contributes primarily to the slow-phase half-life of total testosterone (Fig. 2B). Indeed, the SHBG-bound testosterone half-life was greater than that of total testosterone in 13 of the 14 volunteers. Half-lives of total testosterone were nearly twofold greater than those 
of albumin-bound, bio, and free testosterone. No other published estimates exist for comparison. For the aggregate data, the absolute rank order of decreasing testosterone half-lives was slow-phase SHBG-bound $\mathrm{T}=$ slow-phase total testosterone (any testosterone dose) $>$ slow-phase free, bio, or albumin-bound $\mathrm{T}>$ rapid phase (any testosterone dose and any moiety). One could conjecture that the consistent dose- and moiety-independent rapid-phase half-life of testosterone disappearance during pulsatile testosterone infusions principally reflects initial intravascular distribution of testosterone. In contrast, the nearly tenfold longer slow-phase half-lives of albumin-bound, bio, and free testosterone may mainly reflect rate-limiting loss of testosterone from the bloodstream into interstitial fluids. This postulate remains to be proven directly. Neither SHBG nor albumin need leave the circulation rapidly, given that nominal dissociation half-lives for testosteroneSHBG and testosterone-albumin complexes at $37^{\circ} \mathrm{C}$ are $\leq 12$ and $\leq 0.35$ s respectively, at least in vitro $(14,24$, $27,28)$. Since testosterone bound to SHBG and testosterone bound to albumin may be extracted by certain tissues, the extent to which testosterone must be free or selectively protein-bound to be removed from the circulation may be organ-specific.

The present estimate of the median $V_{\mathrm{d}}$ of total testosterone $\left(25 \mathrm{l} / \mathrm{m}^{2}\right)$ in nine men receiving the two lower doses of testosterone pulses superimposed upon basal testosterone infusion under steroidogenic inhibition compares with a mean value of about $16 \mathrm{l} / \mathrm{m}^{2}$ reported in a study of 5 young men given a single injection of radiolabeled testosterone (16) and about $40 \mathrm{l} / \mathrm{m}^{2}$ in 11 older men given a single 20-min injection of $1 \mu \mathrm{mol}$ testosterone (20). Another analysis of singlebolus tritiated-testosterone decay curves in six men yielded an overall $V_{\mathrm{d}}$ of about $33 \mathrm{l} / \mathrm{m}^{2}$ (29). In marked contrast to total testosterone, estimates of bio testosterone, free testosterone, albumin-bound testosterone, and SHBG-bound testosterone $V_{\mathrm{d}}$ in the present paradigm were $46,501,47$, and $79 \mathrm{l} / \mathrm{m}^{2}$ respectively. To our knowledge, no other published estimates exist for comparison. The apparent $V_{\mathrm{d}}$ of free testosterone was twofold larger than the total body-water space, thus suggesting significant extravascular sequestration. Whereas testosterone exists in plasma as about $2 \%$ free, the remainder is bound to SHBG $(\sim 55 \%)$, albumin $(\sim 50 \%)$, and $\mathrm{CBG}(\sim 3 \%)$. Extravascular tissues putatively sequester testosterone via binding of testosterone to interstitial SHBG, albumin, CBG, and other extraand intercellular proteins, testosterone-transforming enzymes, and androgen receptors (1, 30-32).

By way of caveats, in vivo testosterone-SHBG association and $K_{\mathrm{d}} \mathrm{s}$ are not known. In addition, estimates of in vitro binding constants vary by up to tenfold $(13,14,24,27,28)$. Nonetheless, the facts that in vitro equilibrium dissociation half-times are $12 \mathrm{~s}$ or less, and that dexamethasone (used here as glucocorticoid replacement) does not interfere with
testosterone-SHBG binding, should allow reasonable computational estimates of SHBG-bound, albuminbound, bio, and free testosterone concentrations in each 10-min sample using measured total testosterone, SHBG, and albumin concentrations. For such calculations, SHBG and albumin concentrations were measured every hour during the pulsatile-testosterone clamp, and association constants $\left(1.78 \times 10^{9} \mathrm{M}\right.$ for testosterone-SHBG and $1.80 \times 10^{4} \mathrm{M}$ for testosterone-albumin) were obtained by iterative regression of computation on measured free and bio testosterone concentrations in another cohort of healthy men (Methods). Results of the chemiluminescence testosterone assay were confirmed by sequential LC-MS/MS spectrometry.

Limitations of the present analyses include the need to ultimately measure testosterone concentrations more frequently; extend the range of ages and BMIs evaluated; ascertain in vivo association and dissociation rates of testosterone to and from SHBG and albumin; measure interstitial testosterone concentrations; and compare kinetics of various testosterone-infusion waveforms. One strategy would be to inject fewer testosterone pulses but sample at 2.5- or 5-min intervals to estimate the rapidphase testosterone half-life with greater precision. To the extent that several doses in the current paradigm of pulsatile testosterone delivery mimic episodic testosterone secretion in healthy men, the outcomes presented should apply to the kinetics of testosterone pulses generated endogenously in healthy men of similar age, body composition, and SHBG concentrations.

\section{Supplementary data}

This is linked to the online version of the paper at http://dx.doi.org/ 10.1530/EJE-09-1085.

\section{Declaration of interest}

The authors declare that there is no conflict of interest that could be perceived as prejudicing the impartiality of the research reported.

\section{Funding}

P Y Liu was supported by Career Development Award 511929 from the National Health and Medical Research Council of Australia. Studies were supported in part by the National Center for Research Resources (Rockville, MD, USA) Grant M01 RR00585 to the General Clinical Research Center of the Mayo Clinic and Foundation, and the National Institutes of Health (Bethesda, MD, USA) grants RO1 AG23133 and AG29215, R21 AG23777 and AG31763.

\section{References}

1 Urban RJ, Evans WS, Rogol AD, Kaiser DL, Johnson ML \& Veldhuis JD. Contemporary aspects of discrete peak detection algorithms. I. The paradigm of the luteinizing hormone pulse signal in men. Endocrine Reviews 19889 3-37. 
2 Monet-Kuntz C \& Terqui M. Changes in intratesticular testosterone, cytoplasmic androgen receptors and ABP content of the ram testis after a single endogenous pulse of LH. International Journal of Andrology 19858 129-138.

3 Keenan DM \& Veldhuis JD. Divergent gonadotropin-gonadal doseresponsive coupling in healthy young and aging men. American Journal of Physiology 2004286 R381-R389.

4 Winters SJ \& Troen P. Testosterone and estradiol are co-secreted episodically by the human testis. Journal of Clinical Investigation 198678 870-873.

5 Nankin HR \& Calkins JH. Decreased bioavailable testosterone in aging normal and impotent men. Journal of Clinical Endocrinology and Metabolism $1986 \mathbf{6 3} 1418-1420$.

6 Rowe PH, Racey PA, Lincoln GA, Ellwood M, Lehane J \& Shenton JC. The temporal relationship between the secretion of luteinizing hormone and testosterone in man. Journal of Endocrinology 197564 17-25.

7 Veldhuis JD, King JC, Urban RJ, Rogol AD, Evans WS, Kolp LA \& Johnson ML. Operating characteristics of the male hypothalamopituitary-gonadal axis: pulsatile release of testosterone and follicle-stimulating hormone and their temporal coupling with luteinizing hormone. Journal of Clinical Endocrinology and Metabolism 198765 929-941.

8 Goji K \& Tanikaze S. Spontaneous gonadotropin and testosterone concentration profiles in prepubertal and pubertal boys: temporal relationship between luteinizing hormone and testosterone. Pediatric Research 199334 229-236.

9 Liu PY \& Veldhuis JD. The hypothalamo-pituitary unit, testis and male accessory organs. In Yen and Jaffe's Reproductive Endocrinology: Physiology, Pathophysiology, and Clinical Management, pp 283-298. Eds R Barbieri \& J Strauss. Philadelphia: Elsevier, 2009.

10 Veldhuis JD, Zwart AD \& Iranmanesh A. Neuroendocrine mechanisms by which selective Leydig-cell castration unleashes increased pulsatile LH release in the human: an experimental paradigm of short-term ketoconazole-induced hypoandrogenemia and deconvolution-estimated LH secretory enhancement. American Journal of Physiology 1997272 R464-R474.

11 O'Connor S, Baker HW, Dulmanis A \& Hudson B. The measurement of sex steroid binding globulin by differential ammonium sulphate precipitation. Journal of Steroid Biochemistry 19734 331-339.

12 Singh RJ. Validation of a high throughput method for serum/ plasma testosterone using liquid chromatography-tandem mass spectrometry (LC-MS/MS). Steroids 200873 1339-1344.

13 Takahashi PY, Votruba P, Abu-Rub M, Mielke K \& Veldhuis JD. Age attenuates testosterone secretion driven by amplitude-varying pulses of recombinant human luteinizing hormone during acute gonadotrope inhibition in healthy men. Journal of Clinical Endocrinology and Metabolism 200792 3626-3632.

14 Sodergard R, Backstrom T, Shanbhag V \& Carstensen H. Calculation of free and bound fractions of testosterone and estradiol-17 beta to human plasma proteins at body temperature. Journal of Steroid Biochemistry 198216 801-810.

15 Keenan DM, Roelfsema F, Biermasz N \& Veldhuis JD. Physiological control of pituitary hormone secretory-burst mass, frequency and waveform: a statistical formulation and analysis. American Journal of Physiology 2003285 R664-R673.

16 Horton R, Shinsako J \& Forsham PH. Testosterone production and metabolic clearance rates with volumes of distribution in normal adult men and women. Acta Endocrinologica $1965 \mathbf{4 8}$ $446-458$

17 Akaike H. A new look at the statistical model identification. IEEE Transactions on Automatic Control 197419 716-723.

18 Winer BJ. Statistical Principles in Experimental Design, pp. 232-250, New York: McGraw Hill, 1971.
19 Keenan DM, Alexander SL, Irvine CHG, Clarke IJ, Canny BJ, Scott CJ, Tilbrook AJ, Turner AI \& Veldhuis JD. Reconstruction of in vivo time-evolving neuroendocrine dose-response properties unveils admixed deterministic and stochastic elements. PNAS $20041016740-6745$.

20 White CM, Ferraro-Borgida MJ, Moyna NM, McGill CC, Ahlberg AW, Thompson PD, Chow MS \& Heller GV. The pharmacokinetics of intravenous testosterone in elderly men with coronary artery disease. Journal of Clinical Pharmacology 199838 792-797.

21 Ishimaru T, Edmiston WA, Pages L \& Horton R. Splanchnic extraction and conversion of testosterone and dihydrotestosterone in man. Journal of Clinical Endocrinology and Metabolism $1978 \mathbf{4 6}$ 528-533.

22 Southren AL, Gordon GG \& Tochimoto S. Further study of factors affecting the metabolic clearance rate of testosterone in man. Journal of Clinical Endocrinology and Metabolism $1968 \mathbf{2 8}$ 1105-1112.

23 Schulze JJ, Lundmark J, Garle M, Skilving I, Ekstrom L \& Rane A. Doping test results dependent on genotype of uridine diphosphoglucuronosyl transferase $2 \mathrm{~B} 17$, the major enzyme for testosterone glucuronidation. Journal of Clinical Endocrinology and Metabolism $2008932500-2506$.

24 Nisula BC \& Dunn JF. Measurement of the testosterone binding parameters for both testosterone-estradiol binding globulin and albumin in individual serum samples. Steroids 197934 771-791.

25 Bird CE, Green RN, Calandra RS, Connolly JG \& Clark AF. Kinetics of $3 \mathrm{H}$-testosterone metabolism in patients with carcinoma of the prostate: effects of oestrogen administration. Acta Endocrinologica $197167733-739$.

26 Damassa DA \& Gustafson AW. Effects of chronic infusions of sex steroid-binding protein on the testosterone-mediated inhibition of gonadotropin secretion and maintenance of sex accessory glands in male rats. Endocrinology 1988123 1885-1892.

27 Vigersky RA, Kono S, Sauer M, Lipsett MB \& Loriaux DL. Relative binding of testosterone and estradiol to testosterone-estradiolbinding globulin. Journal of Clinical Endocrinology and Metabolism 197949 899-904.

28 Mendel CM. Rates of dissociation of sex steroid hormones from human sex hormone-binding globulin: a reassessment. Journal of Steroid Biochemistry and Molecular Biology 199037 251-255.

29 Clark AF, Calandra RS \& Bird CE. Kinetics of [3H]-testosterone metabolism in normal young men: effects of medroxyprogesterone acetate (provera) administration. Journal of Steroid Biochemistry $19723837-842$.

30 Hobbs CJ, Jones RE \& Plymate SR. The effects of sex hormone binding globulin (SHBG) on testosterone transport into the cerebrospinal fluid. Journal of Steroid Biochemistry and Molecular Biology 199242 629-635.

31 Krey LC \& McGinnis MY. Time-courses of the appearance/ disappearance of nuclear androgen-receptor complexes in the brain and adenohypophysis following testosterone administration/ withdrawal to castrated male rats: relationships with gonadotropin secretion. Journal of Steroid Biochemistry 199035 403-408.

32 Hammes A, Andreassen TK, Spoelgen R, Raila J, Hubner N, Schulz H, Metzger J, Schweigert FJ, Luppa PB, Nykjaer A \& Willnow TE. Role of endocytosis in cellular uptake of sex steroids. Cell 2005122 751-762.

Received 15 January 2010

Accepted 19 January 2010 